\title{
TV Program Popularity Prediction using Time Base
}

\author{
Tatineni Anusha \\ Student, M.Tech, CSE \\ Gudlavalleru Engineering \\ College \\ Gudlavalleru, India
}

\author{
Garimella Bharathi \\ Assistant Professor, CSE \\ Gudlavalleru Engineering \\ College \\ Gudlavalleru, India
}

\author{
Tatineni Poojitha \\ Assistant Professor, CSE \\ Gudlavalleru Engineering \\ College \\ Gudlavalleru, India
}

\begin{abstract}
The main goal is to the provide better recommendations system to TV viewers here the predictions are based on VOD streaming .and recommender systems have great achievements in the fields of VOD, (VIDEO ON DEMAND). We have provide an effective recommended programs. Based on trending data the rank wiil change based on time series.so that timely prediction of program popularity is of great value for content providers ,advertisers, and broadcast TV operators This information can be beneficial for operators in TV program purchasing decisions and can help advertisers formulate reasonable advertisement investment plans. Prediction models have been proposed based on video-on-demand (VOD) K spectral clustering and Kmedoids with Dynamic time warping are used for popularity prediction .and . file creation with multi grained is used for optimizing the streaming time. Cache replacement strategy is used to free up memory constraints. So it can speed up the streaming data.
\end{abstract}

\section{Keywords}

TV programs, time, context, recommender systems, trending, audience predictionwatch it, regardless to the fact that a more interesting program could be scheduled on a different channel or at a different time slot.

\section{c) A user cannot watch different TV channels simultaneously \\ In non linear TV common recommender systems,}

programs are always accessible and a user can absorbs more content at the simultaneous time, in linear TV a users can look only one program at the particular time and the programs are left are not be convey in

upcoming . consequently, werecognize the viewing

habits, a recommender system should indentify that some

of the TV programs are common because scheduled

simultaneously.

\section{d) Television Audience Ratings}

Television crowd evaluations (TV appraisals) are the major marker in the concept of TV broadcasting; they're utilized to survey the prevalence of TV programs. program's TV rating shows the level of all TV families checked out that program. Television evaluations are a standard measure used to decide the effect of publicizing that is, what number of focused individuals are viewing the shows.

\section{INTRODUCTION}

Since Television has become a part in every one's life.there are number of shows telecasting in different channels. The number o f viewers watching shows are increasing day-by-day. User attention is not distributed among all the programs so predicting
Recommending TV programs is more challenging issues for several aspects:

\section{a) Time-constrained catalog of items}

VOD(video on demand) which provides users with the facility to select and choose content at their convenience, where as in traditional linear TV programs are broadcast according to a preset schedule, and recommendations should consider only programs transmitted at the particular time.

\section{b) TV show patterns:}

Although TV watcher have a number of mechanism to choose . the recommender systems such as linear TV, programs are major important than list of shows. If there is an curiosity of the programs for the end user in her/his like channels and that time slots, the user will

\section{BACKGROUND AND PROBLRM DEFINITION}

The popularity of (HD) and3D increase, IP video will consume large amount amount of traffic over the Internet According to the information the data published by Visual Networking Index in July 2016 [1],the consumption of Internet video streams of broadcast TV will continue to grow .

\subsection{Problem Definition}

since they are number of shows telecasting user concentration is not same for all programs. Only a few programs can attract user attention; the other programs are left without anybody to watch them. Take Tencent video, for instance [2]. Since there have been having almost 45 billion cumulative requests for the top-50 programs, which is more than 80 percent of the total

number of requests. In this only few programs can draw user interest remaining are left over.

\subsection{Problem Statement}

In this scenario it is of great use to predict the popularity of broadcast TV programs using the program popularity prediction results, the user can able to find out the valuable content among the list of programs . programs

or the show which has highest popularity is assigned as the rank 1 .by using this we can identify the top trending show it is done based on the Dynamic time warping .according to the time series analysis the trending and content recommended will automatically change. So

the end user can save much time to find valuable programs among collections of video resources, which will improve user satisfaction and retention. Secondly, based on this program popularity results forecasting data, a company will be able to maximize its advertising 
.we can also identifiy viewing habits of the TV programs and on some characteristic of the TV programs. More specifically, we are interested in recommending some of the pro-grams scheduled to be broadcast within a short time period from the recommendation (e.g., within one hour).

\section{EXISTING SYSTEM}

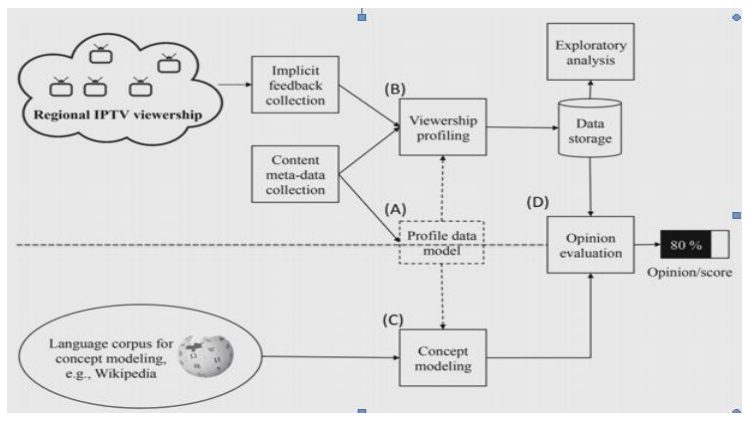

Fig1: Existing System Architecture

The raw input data to the system are

User profiling part of the system. Itis also the basis of the language concepts part of the system as the feature set should present the topic in the best possible way and permit IPTV user profiling to determine user response about the topic of interest. In the scope of an IPTV system the CCE data stream that carries the implicit feedback can be collected in various ways, either on the network or the application level [1]. In either case, the dataset can be viewed as a single anonymzed time-series,, if data is pseudonymized, as a time series per pseudonym Once the time-series data streams are collected, the profiling process can be performed. The goal of the profiling is to quantify viewer reactions to the content shown; depending on the level of anonymization, this can be done on a user-by-user basis or on the population as a whole. The profiling process can in general also take into accountvarious temporal tags (such as time of day, day of week, season) or any provided information about the viewer and their context. However, to gauge public interest and opinion, the proposed framework gives most attention to the data about the content itself. Generalized meta-data can be obtained from the electronic program guides or other forms of content description. However, much more useful in terms of public interest and opinion analysis, and much higher in resolution, is the time-stamped metadata that can be obtained by collecting the closed captions (subtitles).

An emerging market for IPTV. Numerous commercial systems now offer services over the Internet that are similar to traditional over-the cable, or satellite TV. television, time-shifted programming, and content-on demand are all presently available over the Internet. Increased broadband speed will increases, growth ofbroadband subscription base, and Improved video compression technologies have contributed to the emergence of these IPTV services.It is considered that the streaming media will constitute alarge amount of the Internet traffic in theupcomingyears. Among almost all of the methodology used in present days based on multimedia streaming is based on client-server models. Therefore multi- media streamingrequires huge bandwidth, and the server network bandwidth runs out continuously if uncast client-server model is used. Single source multicast is one of the best choice in which that use a single stream model and then it pass it to all the clients. The IP multicast has been slowed by difficult issues related to scalability, and support for higher layer functionality like congestion control and reliability. An IPTV model overcomes the server link bottleneck problem. In the IPTV model, multimediacontents aredeliveredbyusingthebandwidth of the clients themselves.In IPTV streaming networks, users may hop from one channel to another causing traffic loads on the server to buffer up and transmit the selected content. This entireprocess mightimpose a delay $15-30$ sec depending ontraffic conditions and server handling capacity. here wehadimplement a framework called Adaptive ContentInterest Analytics(to combine dynamic groups for Internet streaming. Adaptive Content Interest Analytics supports many groups and can efficiently spread data to these dynamic groups. It first builds a shared overlay Content Interest Analytics for all hosts in the system.

Here our main focus is on content access events (CAE) generated by the viewers. CAE data can be represented by a time series vector; it hides the information about of user behavior and the each content access event is motivated by a combination of viewer's interests and content context. The idea is to identify and demonstrate how the users' interactions with the IPTV service can be efficiently used to gauge the public interest on a specific topic at a large scale.

Nowadays TV viewing has become common along with the help of screens (PC and mobile phone usage) via Cable, Satellite and IPTV are the sources of video Content offered. Internet protocol of Television offers divided into three main groups they are

Live Television, with or without interactivity related to the current TV show;

Time-Shifted Television: catch-up TV (replays a TV show that was broadcast hours or days ago), start-over TV (replays the current TV show from its beginning);

Video on Demand (VOD): browse a catalog of videos, not related to TV programming.

Challenges: Some concepts regarding to TV program popularity are difficult to measure, such as the interests of the audience. Relationship between popular events in the real world and in TV programs cannot be easily introduced into the prediction model, which should be considered when the prediction are based on (K-Spectral) model since it is complicated to process Big Data..So a better system is required to overcome at least some of the challenges.

\subsection{Methods of Existing System}

\subsubsection{K Spectral Clustering}

$\mathrm{k}$ spectral clustering data points are as nodes of a connected graph and clusters are found by partitioning the graph, based on its spectral clustering decomposition, the graph into sub graphs.

- $\quad$ Advantage of $\mathrm{k}$ spectral clustering

It is used to improve recommendations scores.

- Disadvantage of K spectral clustering

$\mathrm{K}$ spectral can not handle large amount of data

It produce in adequate results ,because it does not process the recommendations related to the time .

\subsubsection{KMedoids With Dynamic Time Warping:}

Dynamic Time Warping find the smallest difference between the two time series. There are multiple metrics that could be used to assess how 'similar' two time series are, and some commonly used ones are Euclidean Distance. We apply a dynamic time warping distance based $\mathrm{K}$-medoids algorithm to cluster shows with same popularity This approach is computationally more 
efficient than previous methods DTW distance-based K medoids algorithm is directly driven by raw data. Here we have taken the $\mathrm{k}$ value $=2$ for (activities, , recent activates), such as repeatedly watch, watch later ,add to watch list, skipped and recent activities based up on the time the content recommended will change .according to this it will predict the programs which has the highest score ,highest rank will be pushed as first .and then the next, and this will display the highest overall trending programs.

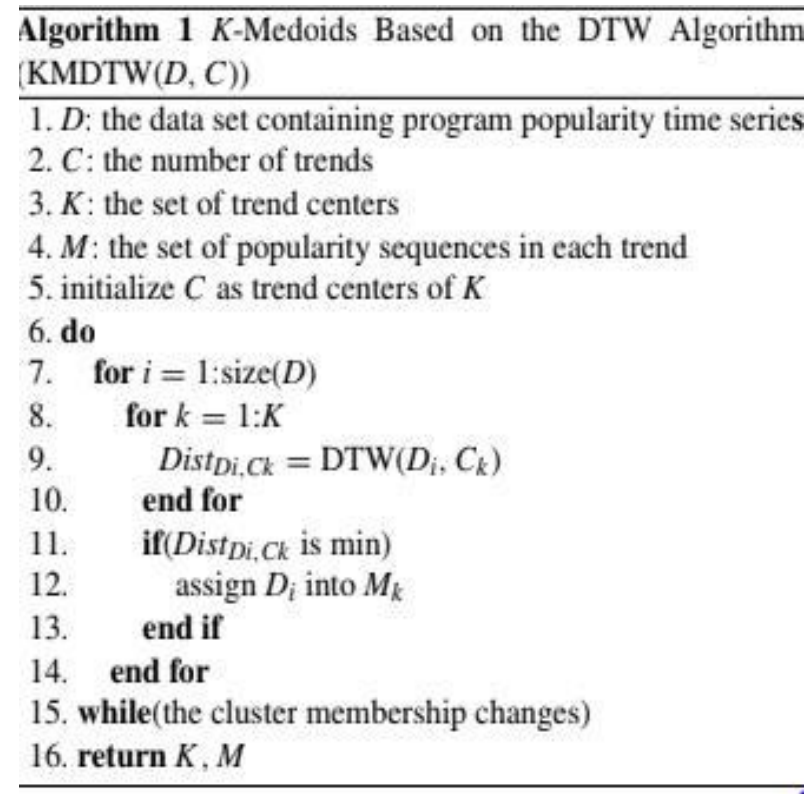

- Disadvantage of Dynamic Time warping:

All the calculations are done in cache memory so buffering time increases, and I take a lot of time

\section{PROPOSED SYSTEM:}

We develop a cache replacement strategy that can proactively adapt to the evolution of program popularity.

- Analysis of Content Popularity Prediction models enables better content recommendations, the IPTV broadcaster is resource constrained due to frequent pagination and caching of content through user activities. We propose to use a cache replacement block management algorithm to free up memory constraints.

- Algorithmic steps are as follows:

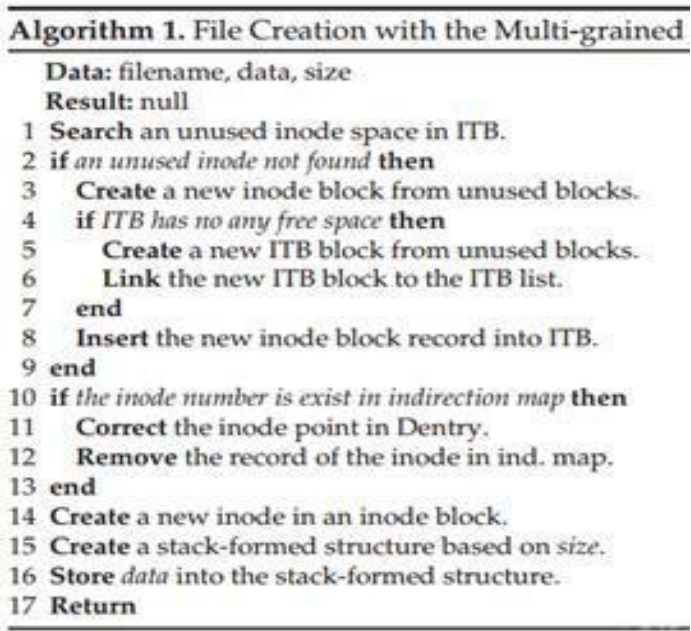

In the proposed cache replacement strategy, all data blocks are reclaimable in the indexed node's translation table(ITB) .

$\square \quad$ The predictive model results are used to determine which frequently requested contents are to be cached and which are to replaced.

$\square \quad$ In the cache reclamation mechanism, when a

content chunks are freed by the movement/deletion of other less frequent one's, the data block can be directly restored.

$\square \quad$ The implemented cache replacement method relies on analyzing frequent file requests logs and predictive model results of popular contents.

$\square \quad$ Advantage of proposed system:

$\square$ Optimized the streaming services in IPTV

environment. With relevant to content recommendation and streaming methods.

\section{EXPERIMENTAL RESULTS}

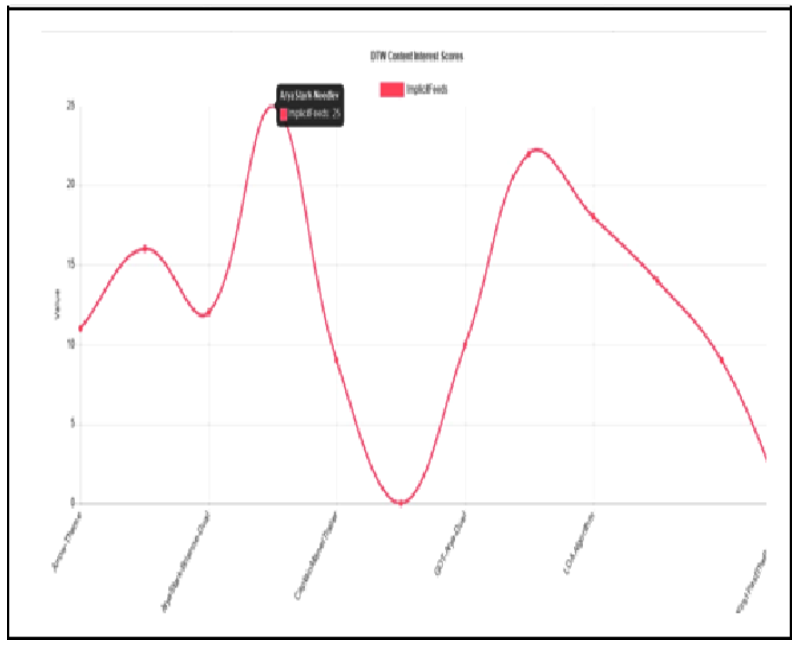

Fig 2. Overall trending score

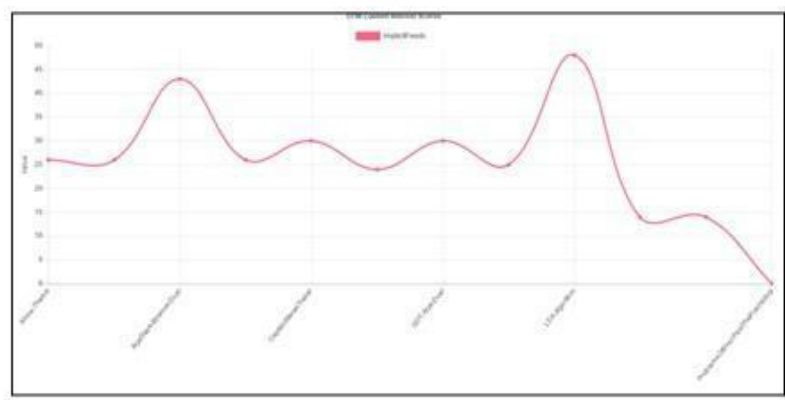

Fig 3. LDA algorithm has overall trending scores

Fig2 and Fig3: shows the over all Trending program..at that particular time..based on the time series, the recommending contents and trending program will updated and the activities of the user is different fromone user to another so the dominate which is having highest score will assign as the over all trending.

\section{CONCLUSION:}

our proposed model uses cache replacement block management algorithm to free up memory constraints in the cache replacement chunks are moved by movement and deletion the implementation of cache replacement method 
improve the accuracy of $20 \%$ and give prediction results much faster.

Performance evaluation

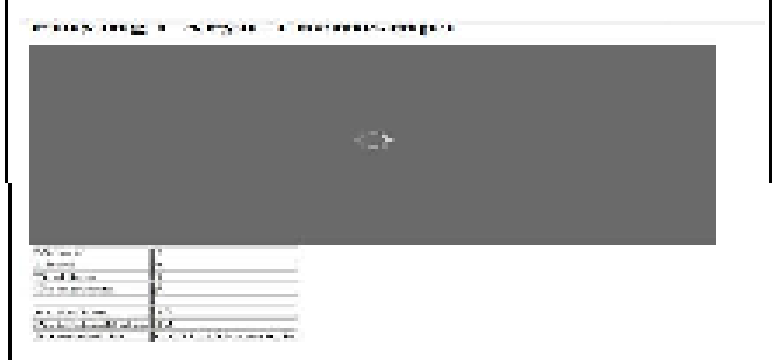

Fig4: optimized the performance of streaming sevices inIPTV enevironment with relevant content recommendations and streaming methods.

\section{REFERENCES}

[1] P.J. Danaher and T.S. Dagger, "Using a NestedLogit Model to Forecast Television Ratings," Int'l J. Forecasting, vol. 28 , no. 3, 2012, pp. 607-622.

[2] P.J. Danaher, T.S. Dagger, and M.S. Smith,"Forecasting Television Ratings," Int'l J. of Forecasting, vol. 27, no. 4, 2011, pp.1215-1240.

[3] H. Yin et al., "A Temporal Context-AwareModel for User Behavior Modeling In Social Media Systems," Proc.
2014 ACM SIGMOD Int'l Conf . Management of Data (SIGMOD), 2014, pp. 1543-1554.

[4] Ferraz Costa et al., "RSC: Mining and Modeling Temporal Activity in Social Media," Proc. 21th ACM SIGKDD Int'l Conf. Knowledge Discovery and DataMining (SIGKDD), 2015, pp. 269-278.

[5] [5] R. Hinami and S. Satoh, "Audience Behavior Mining by Integrating TV Ratings with Multimedia Contents," IEEE Int'l Symp. Multimedia (ISM), 2016, pp. 44-51.

[6] U. Sedlar, M. Volk, J. Sterle, A. Kos, and R.Sernec, "Contextualized monitoring and root cause discovery in IPTV systems using datavisualization," IEEENetwork, vol. 26 , no. 6 , pp. 40-46, 2012.

[7] A. Tatar, P. Antoniadis, M. D. de Amorim, and S. Fdida, "From popularity prediction to ranking online news," Social Netw. Anal. Mining, vol. 4,pp. 174-183, Dec. 2014.

[8] H. Pinto, J. M. Almeida, and M. A. Gonç alves," "Using early view patterns to predict the popularity of youtube videos," in Proc. 6th ACM Int. Conf.Web Search Data Mining, 2013, pp. 365-374.

[9] A. Kaltenbrunner, V. Gómez, and V. López, "Description and prediction of slashdot activity," inProc. Latin Amer. Web Conf. (LA-WEB), 2007,pp. 57-66. 